\title{
Análisis de la implementación de los espacios de aprendizaje en el aula de Educación Física Infantil \\ Analysis of the implementation of learning environments in the Early Childhood Physical Education class
}

\author{
Cecilia Nicolás Belmonte y José Ignacio Alonso Roque
}

Universidad de Murcia (España)

\begin{abstract}
Resumen. Los espacios de aprendizaje permiten una enseñanza basada en una relación menos estructurada con los materiales, el espacio, los compañeros y los docentes. En esta investigación de carácter cualitativo se hace uso de estos espacios de aprendizaje; en concreto de los ambientes y los rincones de aprendizaje para estudiar dos objetivos: (a) analizar el pensamiento docente sobre la implementación de los espacios de aprendizaje y su incidencia en el proceso de aprendizaje durante las sesiones de Educación Física Infantil, y (b) examinar la repercusión de los espacios de aprendizaje sobre la práctica docente: ideas de las maestras, sentimientos generados, esfuerzo realizado, y propuestas de cambio o mejora, entre otros. Los participantes de este estudio fueron tres maestras de Educación Infantil. Las técnicas e instrumentos empleados fueron la observación participante, un diario de campo, entrevistas individuales y un grupo de discusión. Una vez analizados los datos con Atlas. Ti v 7, los resultados apuntan a unos datos favorables en el uso de los espacios de aprendizaje, con cambios en el pensamiento y la práctica docente. Como conclusión se indica que los espacios de aprendizaje generan cambios en el pensamiento docente en relación a esta metodología y sobre la práctica docente de los mismos.
\end{abstract}

Palabras clave. Aprendizaje activo, Educación Infantil, Educación Física, investigación empírica, observación, registros de investigación, entrevistas, discusión (método pedagógico).

\begin{abstract}
Learning environments allow a teaching based on a less structured relationship with materials, spaces, peers, and teachers. This qualitative research makes use of learning environments; in particular of learning environments and learning corners to study two objectives: (a) analyze the teacher's thoughts about the implementation of learning environments and its impact on the learning process in a Early Childhood Physical Education class, and (b) examine the impact of learning environments on teaching practice: ideas from teachers, feelings generated, efforts made, and proposals for change or improvement, among others. Participants in this study were three Early Childhood Education teachers. Techniques and instruments used in the data collection were participant observation, a field diary, individual interviews and a group discussion. After analyzing the data with Atlas.Ti v7, the results point to favorable data on the use of learning spaces, with changes in teacher's thoughts and even in their teaching practice. As a conclusion it is indicated that learning environments generate changes in the teacher's thoughts about this methodology and about their own teaching practice
\end{abstract}

Keywords. Experiential learning, Early Childhood Education, Physical Education, empirical research, observation, research registers, interviews, discussions (teaching method).

\section{Introducción}

Imaginemos que podemos observar sin que nadie nos vea el desarrollo completo de una clase de Educación Física (EF en adelante) en Educación Infantil (EI), ¿qué es lo que vemos? Probablemente ya estemos visualizando a un gran grupo de alumnos entrando a un pabellón de la mano de un docente, sentándose en círculo en el suelo y esperando las instrucciones del maestro para ir realizando las actividades o juegos previstos. Además, entendemos que estas actividades y juegos persiguen un enfoque globalizado con el objetivo de

Fecha recepción: 01-12-20. Fecha de aceptación: 31-05-21

Cecilia Nicolás Belmonte

cecilia.nicolas@um.es promover el desarrollo integral del alumno. Una vez explicadas estas actividades podemos ver a los alumnos realizar el primer juego y al docente explicar y realizar el segundo, explicar el tercero, etc., y así hasta que termina la clase. Explicación, realización, explicación, realización. Las interacciones entre docentes y alumnos en las sesiones de EF pueden ser así, pero, ¿solo existe este tipo de relación? ¿Se podría conseguir el desarrollo de sesiones de EF con el mismo grado de globalización y enfoque interdisciplinar sin necesidad de este constante intercambio?

En esta investigación se apuesta por el uso de metodologías basadas en los espacios de aprendizaje (EA en adelante), donde las interacciones entre docentes y alumnos son diferentes en relación al tiempo, no al modo, y con las que se puede generar el mismo grado 
de globalización e interdisciplinariedad que en las sesiones convencionales, como la descrita anteriormente. Los espacios de aprendizaje que aquí se mencionan se entienden como espacios físicos con gran cantidad de oportunidades de enseñanza y aprendizaje, ya que el alumnado puede aprender en cualquier lugar (Abbott, 2015). No obstante, hablar de espacios de aprendizaje también es hablar de lugares que generan el ambiente y tiempo idóneo para la relación social de las personas que se encuentran en ellos y para la proliferación de experiencias lúdicas ajustadas a la realidad que las envuelve. Como señala Abott (2015), los docentes han de programar e implementar estos espacios, pero para poder conseguir realmente los objetivos previstos es necesario aceptar cambios y modificaciones en ellos, a corto y largo plazo. Además, los docentes se encuentran con tres desafíos en el momento de diseñar y aplicar los EA. Por un lado el de saber cómo combinar la pedagogía llevada a cabo con el diseño de los mismos. Por otro lado el de crearlos lo más flexibles y sostenibles posible para que se adapten a las necesidades del alumnado y del proceso de enseñanza-aprendizaje.Y finalmente, el tercer desafío es el de saber combinar los espacios físicos con los virtuales, o en este caso, saber diseñar espacios físicos que por sí mismos inviten al aprendizaje (Abbott, 2015).

\section{Los espacios de aprendizaje como espacios interactivos}

Cuando en esta investigación se habla de EA, se hace alusión tanto a los ambientes de aprendizaje (AA en adelante), como a los rincones de aprendizaje (RA). Estos espacios de aprendizaje favorecen en el alumnado el aprendizaje a través del juego, lo que contrasta con la idea expresada por Bássoli et al. (2021) de que «los niños quieren moverse, jugar y aprender jugando.» (p. 842)

Los AA son espacios que han sido preparados y planificados en función de un objetivo concreto y que, debido a su organización, atraen por sí mismos la atención de los alumnos para que los usen y empleen los materiales que hay en ellos (Blández, 2000). Además es importante destacar que la práctica motriz de los alumnos provoca mejoras en la manera de ocupar el espacio, «pasando de ocuparlo solo con el cuerpo, a utilizarlo para construir y delimitar espacial y temporalmente» (Miraflores \& Goldaracena, 2020, p. 626).

En los AA los niños son los protagonistas absolutos, ya que a través de la acción libre y espontánea van adquiriendo el conocimiento. El rol del maestro en esta metodología es el de orientar la sesión. La flexibilidad que existe en los AA es señalada por Smith y Pellegrini (2013) como uno de los aspectos más importantes del juego, al igual que el medio en el que se desenvuelven (y no la finalidad) y el afecto positivo que se transmite. Los AA proporcionan la libertad de elección y el movimiento necesario en las edades tempranas, ya que es necesario que exista un tiempo destinado al «juego en el cual el niño pone sus propias reglas, el tiempo de inicio y el fin de cada juego creado, y pulsa el botón de arranque del mecanismo de la creatividad.» (Arufe, 2020, p. 593).

Se han encontrado diferentes estudios y experiencias educativas con los AA en los que resalta la repercusión positiva que estos tienen sobre el alumnado (Ates, 2013; Barrett, Davies, Zhang \& L, 2015; Hyndman, Kevitt \& Mc, 2012; Rachel, Nnamdi \& Thomas, 2016; Rimm-Kaufman \& Sandilos, 2015; Uncapher, 2016). Encontramos estudios relacionados con los ambientes de aprendizaje, como los de Navarro y García (2010) o Fajardo y García (2010), que señalan que el uso de estos en las sesiones de EF Infantil tiene resultados positivos tanto en docentes como en alumnos. Por este motivo se hace aún más necesario que exista un cambio docente, en la que los maestros adapten su metodología hacia la inclusión de este tipo de espacios para mejorar con ellos el aprendizaje del alumnado y la adquisición y desarrollo de sus capacidades (Blackmore, Bateman, Loughlin, O’Mara \& Aranda, 2011; Walker, Brooks \& Baepler, 2011). Además, se espera que los AA favorezcan el desarrollo de las habilidades motrices básicas, así como de la espontaneidad, la participación en clase, creatividad y capacidades expresivas del alumnado (Hernández et al., 2020).

Por otro lado, para poder definir lo que son los RA es necesario pensar en ellos como los espacios que hay en el aula ordinaria, que sirven para trabajar los diferentes contenidos educativos (Montero, 2012; Vicente, López \& Vallés, 2014). Los RA empleados en EF han de explicarse al inicio de la sesión. En cada rincón tiene que haber uno o varios juegos o actividades que el alumnado ha de realizar cada vez que se encuentra en él. Estos espacios han de estar bien señalizados y delimitados espacialmente para evitar confusiones con otros espacios, y por tanto con la realización de otras actividades. Por su parte, Duncan y Arnott (2019) indican que los juegos se han de seleccionar teniendo en cuenta aspectos como las interacciones interpersonales que pueden resultar, o el material que se va a utilizar, organizando estas variables para que las actividades realizadas 
potencien el juego creativo. Gracias a la actividad física que generan este tipo de espacios, se consigue alcanzar más fácilmente el aprendizaje en el alumnado (Nielsen, Romance \& Chinchilla, 2020).

En definitiva, en los RA el alumnado realiza diferentes actividades lúdicas que les suponen un reto siempre alcanzable, lo que fomenta la motivación en ellos, además, este tipo de juego no promueve la competitividad y es óptimo para generar una reflexión final de todo lo realizado (García \& Alarcón, 2011).

\section{La conducta motriz, núcleo de la enseñanza}

El juego en las edades tempranas es algo común y universal para todos los niños. Además es susceptible de ser empleado como la actividad principal en el proceso de aprendizaje (Gil-Espinosa et al., 2018).

Autores como Parlebas (2001,p.276) lo definen como una «situación motriz de enfrentamiento codificado (...). Cada juego se define por un sistema de reglas que determina su lógica interna». Para Burghardt (2014) el juego es indispensable en el desarrollo del alumnado, puesto que incide sobre el desarrollo de la personalidad del mismo, de hecho, privar a los niños de este puede traer consecuencias negativas en aspectos como la socialización, la salud mental y el desarrollo emocional (Brown, 2014). Según Meneses y Monge (2001) los niños juegan para poder desarrollar su personalidad, comunicarse, estar en contacto con su propio cuerpo y con otras personas y objetos, para fantasear libremente a la vez que se divierten y para desarrollar habilidades. Por estas razones el juego se debe planificar y organizar para poder dotarlo de significatividad educativa haciendo uso de la conducta motriz.

Según Lagardera y Masciano (2014) la conducta motriz es el comportamiento motor asociado a una persona en concreto y abarcando su forma de actuar, sus emociones, sentimientos, motivaciones y deseos. Estos autores señalan que mediante las conductas motrices las personas pueden expresar su forma de sentir y entender la vida, de manera consciente o inconsciente. El estudio realizado por Adolph y Franchak (2016) indica que los comportamientos motores del alumnado, y por tanto el desarrollo de su conducta motriz, están influenciados por la vinculación con los otros durante los tiempos de juego y el entorno que rodea estas situaciones.

Cuando «se educan las conductas motrices se actúa pedagógicamente sobre la unidad del ser» (Lagardera \& Lavega, 2011, p.28). Basándose en su investigación, Lagardera y Lavega (2011, p.40) señalan que una educación física centrada en la persona que aprende, en la pedagogía de las conductas motrices, ayudaría a optimizar las conductas motrices en un marco de acción, pero también a generar una infraestructura motriz que facilitaría cada día las funciones cartográficas del cerebro, y como consecuencia, la mejora ostensible de la calidad de vida, tanto sensitiva, como motriz e intelectual.

Lagunas y preguntas de investigación en los espacios de aprendizaje

Los EA, en este caso los AA y RA, son óptimos para su uso en los procesos educativos, pero ¿cómo son entendidos por los docentes? Hay varias lagunas en el empleo de estos EA, ya que se suele confundir metodológicamente el uso de los RA con el de los AA. También hay ocasiones en las que se confunde el uso de los EA con el juego libre y no estructurado, además el profesorado tiende a emplear los EA en los tiempos libres, por lo que no hay supervisión de lo que realizan los alumnos. Por otro lado, diversos estudios como los de Jung (2011), Moreno, López, Gutiérrez, Cascada y Fernández (2004) y Sáenz-López (1999), indican que sigue habiendo casos en los que los docentes no prestan la suficiente atención a la planificación de la EF, otorgándole un papel secundario a la misma, por lo que en consecuencia no se hace uso de estas metodologías basadas en espacio y la motricidad para generar el aprendizaje.

Por todo lo anterior, se establecen las siguientes preguntas de investigación:

a. ¿De qué manera inciden los EA en el proceso educativo y en la adquisición del conocimiento en el alumnado?

b. ¿Cómo pueden los EAfavorecer el desarrollo global del alumnado?

c. ¿Cómo pueden los EA favorecer el aprendizaje autónomo del alumnado?

d. ¿Qué repercusión pueden tener los EA en la práctica docente y en la manera de orientar las sesiones de Educación Física Infantil?

\section{Objetivos}

Para poder dar respuesta a las lagunas encontradas y a las preguntas de investigación, en este estudio se establecieron los siguientes objetivos:

1. Analizar el pensamiento docente sobre la implementación de los espacios de aprendizaje y su incidencia en el proceso de aprendizaje durante las sesiones de Educación Física Infantil. 
2. Examinar la repercusión de los espacios de aprendizaje sobre la práctica docente: ideas de las maestras, sentimientos generados, esfuerzo realizado, y propuestas de cambio o mejora, entre otros.

\section{Material y método}

\section{Diseño de investigación}

Esta investigación es de carácter cualitativo (Bisquerra, 2004), ya que se acerca a la realidad con el objetivo de comprender fenómenos educativos con el alumnado, cuestionar los comportamientos personales tanto de los alumnos como de los docentes y abordar la realidad como algo holístico. Por otro lado, también se puede indicar que esta investigación consiste en el estudio de un caso típico (McMillan \& Schumacher, 2011) y único, ya que en ella se analiza el proceso y las consecuencias de un programa de intervención en un contexto y con unos participantes determinados. Por este motivo, conviene señalar que todos los resultados obtenidos, así como el análisis que se hace de ellos, parten de las opiniones de las docentes que participan en esta investigación. No se puede realizar una generalización de estos resultados, así como tampoco extrapolar a otros contextos lo que aquí se expone.

Por otro lado, este estudio posee un diseño emergente ya que las decisiones tomadas dependen de la información previa que se posee, y la obtención de datos y su análisis pueden tener momentos simultáneos, puesto que no es necesario que todo sea secuencial.

La investigación cualitativa se basa principalmente en una concepción de la realidad como algo heterogéneo, social e interactivo, que se ocupa de entender los fenómenos sociales desde el punto de vista de los participantes a través de diferentes métodos y estrategias interactivas, como las entrevistas o la observación participante, técnicas que se emplean en el presente estudio (McMillan \& Schumacher, 2011). Por este motivo, el papel que desarrollan los investigadores es el de observar los comportamientos y actitudes de los participantes, interaccionando con ellos y manteniendo una actitud imparcial.

\section{Contexto y participantes}

La investigación se realizó en un centro de EI y Educación Primaria de carácter público del municipio de Murcia. En cada uno de estos cursos de EI se imparte psicomotricidad o EF Infantil.

En relación a los participantes de este estudio, estos se eligieron según el principio de pertinencia (Bisquerra,
2004), es decir, buscando a aquellos que más información y de mayor calidad podían aportar en el tema de investigación. Por este motivo, la búsqueda de participantes se realizó atendiendo a dos criterios de inclusión principales:

1. Que fuesen docentes especialistas de EI.

2. Que fuesen ellos los que impartieran EF Infantil.

La búsqueda de estos maestros se realizó en cuatro centros educativos. Finalmente, en el centro educativo donde se desarrolló esta investigación, se contó con la aceptación del equipo directivo y con la aceptación de tres maestras, que además cumplían los criterios señalados.

Una vez que se contaba con la aceptación del equipo directivo y de las maestras, se procedió a enviar una carta de presentación del investigador a la dirección del centro y una hoja de consentimiento informado a las docentes, en la que se expresaba entre otras cosas, que estaban de acuerdo en participar en dicho estudio.

Por su parte, las tres participantes contaban con más de 25 años de experiencia, y dos de ellas llevan en este mismo centro escolar desde sus inicios en la profesión.

Cada una de las tres participantes se correspondían con las tres edades de EI, una de ellas era participante con un grupo de veinticinco alumnos de tres años de edad, otra era participante con un grupo de veintitrés alumnos de cuatro años de edad, y la tercera y última participante era la tutora de un grupo de veinticinco alumnos de cinco años de edad.

Para establecer un clima de confianza del investigador con los docentes y el alumnado, el investigador acudió en varias ocasiones al centro antes de comenzar con el programa de intervención. Así mismo, antes de comenzar la puesta en práctica de este programa se explicó al alumnado lo que se iba a realizar y se le dio normalidad al hecho de que hubiese una persona más en el aula, con el objetivo de que ellos actuasen como de costumbre.

\section{Instrumentos de recogida de información}

En esta investigación se han empleado los siguientes instrumentos de recogida de información:

A. Observación participante. La observación participante es una de las principales estrategias multimétodo de recogida de información en la metodología cualitativa (McMillan \& Schumacher, 2011). Con el uso de este instrumento el investigador estuvo presente físicamente en el momento en el que se realizaron todas las acciones que se querían observar. No obs- 
tante, la intervención activa del investigador durante las clases fue reducida, ya que solo intervino en aquellos momentos en los que necesariamente tenía que hacerlo, por lo que su grado de participación fue limitado (Bisquerra, 2004).

B. Diario de campo. De la observación participante surgió un diario de campo (Velasco \& Díaz de Rada, 2006). En este diario de campo se realizó un registro continuo de los acontecimientos y actividades que se realizaron, la formulación de nuevos proyectos o actividades que surgieron, los comentarios realizados, y las necesidades y resultados presentados durante el proceso. Este registro no contó con detalles generales sino con información específica y concreta, aquella que podía dar respuesta a los objetivos planteados. Su elaboración se realizó fuera del escenario de investigación, cuando se pudo hacer una reflexión conjunta de todos los aspectos o acontecimientos vividos durante los encuentros con los participantes.

C.Entrevistas. Las entrevistas utilizadas fueron semiestructuradas (Bisquerra, 2004) o guiadas (McMillan \& Schumacher, 2011), de respuesta abierta y caracterizadas por su flexibilidad en cuanto a su formulación y respuesta. En este tipo de entrevistas los temas de los que se querían hablar se preestablecieron antes de la conversación, pero no fueron inamovibles. Entre las preguntas realizadas en estas entrevistas se encuentran algunas relacionadas con la experiencia docente, con las opiniones y valores en relación a la docencia y con los sentimientos durante la realización de este estudio.

Estas entrevistas tuvieron el objetivo de conocer cómo se sintieron las maestras, qué opinaban respecto a los EA y saber cómo fue el desarrollo de la intervención educativa, para ello se realizaron tres entrevistas diferentes a cada maestra. Con la entrevista inicial se pretendía crear un acercamiento con las docentes y conocer su labor en el aula ordinaria en las sesiones específicas de EF. La segunda entrevista tenía la finalidad de conocer cómo se sentían y qué pensaban de las metodologías que hasta el momento se habían desarrollado. Mientras que la última entrevista pretendía obtener información acerca de sus sentimientos, vivencia y evaluación personal durante el desarrollo de las diferentes sesiones, así como sus pensamientos y propuestas de cambio, mejora o permanencia.

Estas entrevistas fueron validadas por dos profesionales del ámbito de la didáctica general y por otros dos profesionales del ámbito de la EF. A estos profesionales se les ofreció una tabla inicial de validación, modificada de la propuesta por Corral (2009). En esa tabla se vali- daron diferentes aspectos, (a) claridad en la redacción de la pregunta, (b) coherencia de la pregunta con el tema de investigación, (c) introducción de la pregunta a la respuesta, (d) lenguaje adecuado con el nivel del informante $y$, finalmente, (e) conocer si las preguntas permitían acceder a la respuesta deseada.

La escala de puntuación para las preguntas en esta tabla de validación fue: 1. muy mala, 2. mala, 3. regular, 4. buena y 5 . muy buena.

La puntuación y comentarios referidos en cada uno de los apartados fueron analizados y valorados teniendo en cuenta los siguientes criterios:

1. Cuando dos o más aspectos tuvieron un tres, o menor puntuación, se modificó la pregunta.

2. Siempre que hubo una puntuación de uno se revisó la pregunta, no significando esto que finalmente se modificase la misma.

3. El criterio número dos se anuló si dos o más puntuaciones eran de cinco.

4. Incluso aunque una pregunta no necesitase modificación, en algunas ocasiones se optó por modificarla según algunas sugerencias cualitativas.

Estas entrevistas fueron grabadas para poder hacer una transcripción posterior de las mismas y facilitar su proceso de análisis. El proceso de transcripción se llevó a cabo de forma manual, lo que sirvió a su vez para una mejor asimilación de los datos obtenidos.

D. Grupo de discusión. El grupo de discusión de esta investigación estuvo formado por las maestras participantes. Las dimensiones tratadas durante el mismo estaban relacionadas con aspectos socioafectivos, cognitivos y motrices.Los temas que se debatieron estaban vinculados a la puesta en práctica de los diferentes espacios de aprendizaje en EF y lo que esto significó para ellas: esfuerzo, sentimientos, opiniones, ideas y propuestas de cambio o mejora.

El investigador fue quien guió los diálogos entre las docentes y quien encauzó los temas tratados, interviniendo en aquellos casos en los que se podía enriquecer el diálogo, por lo que se trató de una conversación libre pero moderada. Para poder generar ese sentimiento de libertad fue necesario crear previamente un clima de confianza, tranquilidad y comodidad.

$\mathrm{Al}$ igual que en el caso de las entrevistas realizadas, la información que se trató durante el grupo de discusión fue grabada y posteriormente transcrita manualmente.

\section{Procedimiento de actuación}

Se elaboraron seis sesiones, tres de ellas de AA de- 
finidos, y las otras tres para los RA. En cada sesión se crearon cuatro ambientes diferentes o cuatro rincones diferentes, por lo que en total se diseñaron doce AA y otros doce RA. No obstante, algunos de los ambientes o rincones presentaron similitud entre ellos, ya que en ocasiones solamente se añadía complejidad al EA en función de la edad del alumnado.

Como puede verse en la Figura 1, todas las sesiones duraron aproximadamente una hora y se diseñaron siguiendo la estructura propuesta por López-Pastor (2006):

1. Asamblea inicial: la que cada maestra realizaba habitualmente, es decir, calentamiento corporal inicial, explicación general de la sesión y recordatorio de las normas de comportamiento. (10 min.)

2. Parte principal de la actividad motriz. (40 min.)

3. Asamblea final, donde se incluyó la relajación y la verbalización de lo realizado. (10 min.)

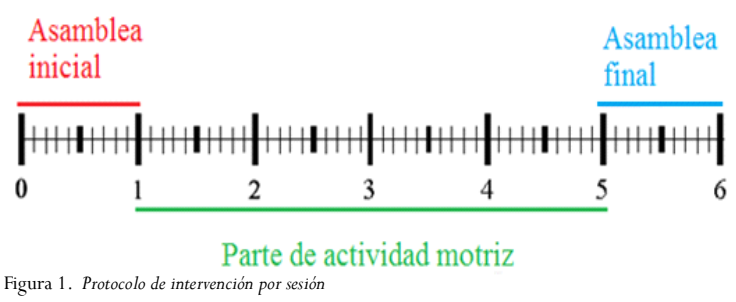

Se procuró que cada vez que el alumnado entrase a la sala donde se realizaban estas sesiones, esta estuviera preparada y con todo el material expuesto para que hubiera gran impresión y motivación, tanto para el alumnado como para las maestras. Se intentó que la sala siempre contara con buena luz solar, por lo que se tenían todas las persianas subidas, y también se intentaba que no hubiera ruidos externos que entorpecieran la atención del alumnado y concentración de las docentes. Una vez que los niños entraban en la sala, estos se sentaban en el suelo y la maestra comenzaba con la asamblea inicial y la explicación de lo que se iba a realizar durante esa sesión. Cuando ya estaban dadas las consignas de lo que se tenía que hacer y se había hecho una ejemplificación de cada actividad con los niños (en el caso de los RA), se procedía a comenzar con la parte principal de la actividad motriz. Durante este momento, tanto las docentes como la investigadora tenían una actitud de observación y escucha activa, ya que de esto derivó una parte de la evaluación del proceso de enseñanza-aprendizaje de los EA. Las maestras también recordaban de vez en cuando las normas de la sesión y la manera de realizar cada juego o actividad, y la investigadora mantenía un rol de observadora participante, involucrándose en el desarrollo de la sesión solo cuando la situación lo requería.
Para la selección de las actividades realizadas en cada sesión se tuvieron en cuenta los siguientes factores:
a. La edad del alumnado.
b. El espacio y el tiempo disponible.
c. El material disponible.
d. Que todo el alumnado pudiese realizarlas.

\section{Análisis de la información}

La información recogida se analizó con el programa ATLAS. Ti. v. 7.0. Con sus herramientas se pudo gestionar, ordenar y reagrupar el material con el que se trabajó. Se establecieron códigos deductivos y se generaron otros de carácter inductivo. Dado que se observó que en ocasiones los dos tipos de códigos se daban en las mismas citas de los participantes, se procedió a realizar un análisis de coocurrencia de los mismos; y posteriormente, se generaron dos redes semánticas con los principales códigos deductivos preestablecidos. Todos estos códigos fueron obtenidos de los documentos primarios de información con los que se trabajó: entrevistas en profundidad, grupo de discusión y diario del investigador. El proceso que se siguió para el análisis de estos documentos fue el que McMillan y Schumacher (2011) propusieron en el sistema de organización a partir de los datos:

1. Primero se leyeron todos los datos obtenidos y se fueron apuntando las ideas que destacaban de cada uno de ellos.

2. Después se establecieron los principales temas que se trataban.

3. Tras esto se realizó una comparación de temas, para evitar que hubiera algunos muy similares entre sí.

4. Seguido de esto se conectaron diferentes temas entre sí en función de sus similitudes y diferencias.

5. Por último, se realizó un perfeccionamiento de toda esta organización, revisando todos los temas obtenidos e intentando que quedase un número asequible de temas con los que poder trabajar y manejar sin grandes problemas.

El análisis se realizó generando cuatro unidades hermenéuticas referidas a las entrevistas iniciales, las continuas, las finales, el diario de campo del investigador y el grupo de discusión.

\section{Resultados}

En lo referente al primer objetivo, «analizar el pensamiento docente sobre la implementación de los espacios de aprendizaje y su incidencia en el proceso de aprendizaje durante el desarrollo de las sesiones de Educa- 
ción Física Infantil», se parte de lo señalado en la Figura 2.

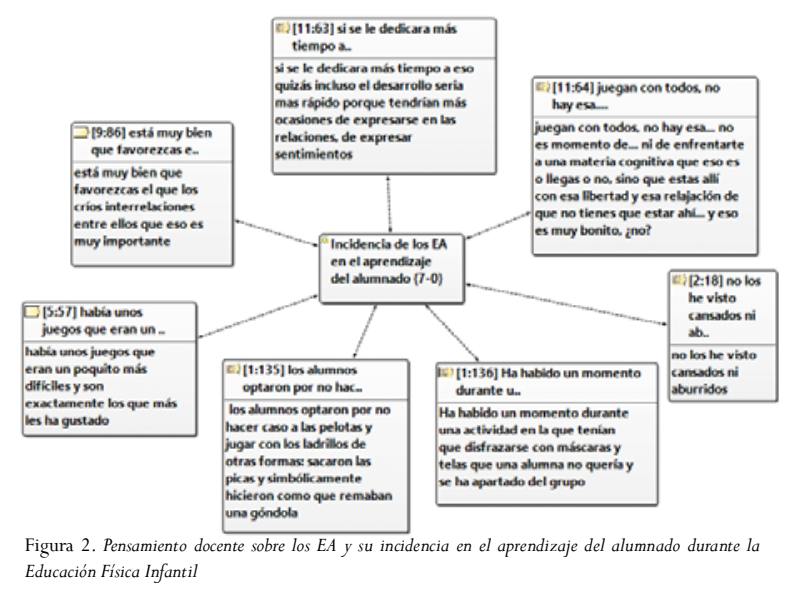

Según la interpretación y el pensamiento de las docentes, la incidencia del uso de los EA en el proceso de aprendizaje del alumnado va aparejado con diversos aspectos a tener en cuenta. Por un lado se percibe que el desarrollo de las sesiones de EF basadas en la implementación de los EA destaca positivamente sobre todo el alumnado, incluyendo el Alumnado con Necesidades Específicas de Apoyo Educativo (ACNEAE). Las docentes señalan que eso se debe a que el uso de los EA en las sesiones de EF Infantil permiten superar las barreras cognitivas e ir más allá, centrándose en otros aspectos más emocionales o sociales, como se puede observar en la Figura 2: «juegan con todos (...) no es momento de enfrentarse a una materia cognitiva, que eso es llegas o no, sino que estás allí, con esa libertad y esa relajación...» [Maestra 2, Grupo de discusión]

Por otro lado, las docentes indican queuna de las ventajas de los EA es poder plantear juegos con un nivel de dificultad elevado. Estos juegos generan un nivel de motivación mayor en el alumnado porque no siempre está la figura del docente dirigiendo o salvando al alumno para superar la acción, es decir, cuando lo consiguen, lo hacen por ellos mismos. Por este motivo este tipo de juegos son los que más les gustan y en los que más ilusión y esfuerzo ponen (Figura 2).

En relación al estado emocional del alumnado, las maestras han percibido que no han notado aburrimiento o desidia en ellos: «no los he visto ni cansados ni aburridos» durante las sesiones de EF Infantil [Maestra 1, entrevista continua]. No obstante, en la Figura 2 también se observa que el estado emocional del alumnado no siempre ha estado vinculado a emociones positivas, ya que hubo una niña que no llegó a disfrazarse en una actividad que lo requería porque tenía miedo de los disfraces, lo que la hizo alejarse de esa zona y jugar en otros espacios.

Además de todo lo anterior, las docentes piensan que «si se le dedicara más tiempo a eso - EF- quizá incluso hasta el desarrollo del alumnado sería más rápido porque tendrían más ocasiones de expresarse en las relaciones, de expresar sentimientos.» [Maestra 3, Grupo de discusión]

En la Figura 2, también se observa que los docentes relacionan los EA con las relaciones interpersonales que estos son capaces de generar en el alumnado. Señalan además que la actitud de los alumnos ante los EA es positiva y enriquecedora ya que se aprecia cómo el alumnado desarrolla el juego simbólico mediante estas metodologías: «los alumnos optaron por no hacer caso a las pelotas y jugar con los ladrillos de otras formas: sacaron las picas y, simbólicamente, hicieron como que remaban una góndola» [Diario del investigador].

Por otro lado, en la Figura 3 se puede ver lo más significativo en relación al segundo objetivo, «examinar la repercusión de los espacios de aprendizaje sobre la práctica docente: ideas de las maestras, sentimientos generados, esfuerzo realizado, y propuestas de cambio o mejora, entre otros».

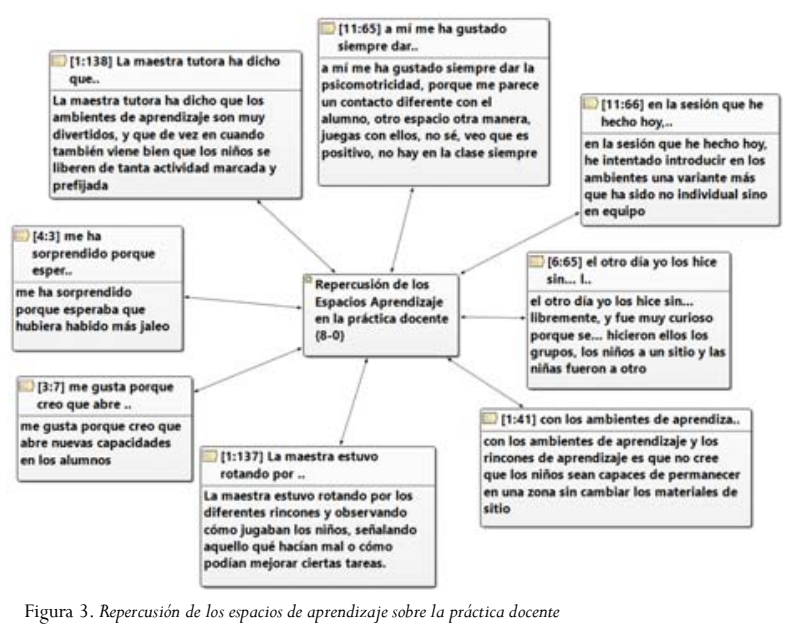

En la Figura 3 puede observarse que los docentes pensaban que las actividades y los juegos que se derivan de los EA, es decir, de los ambientes y los rincones, generarían más descontrol en el aula: «no creo que los niños sean capaces de permanecer en una zona sin cambiar los materiales de sitio» [Diario del investigador].

Por otro lado, las maestras consideran «que los AA son muy divertidos, y que de vez en cuando también viene bien que los niños se liberen de tanta actividad marcada y prefijada» (Figura 3) [Diario del investigador].

Las docentes también señalaron que llevaron a cabo estas metodologías en sesiones diferentes a las realiza- 
das durante este estudio. Indicaron que las realizaron conalgunas variantes, como por ejemplo la de modificar los agrupamientos. Las docentes también comprobaron que esta manera de presentar la EF al alumnado tiene efectos «muy curiosos», ya que «hicieron ellos solos los grupos, los niños - se fueron- a un sitio y las niñas fueron a otro» [Maestra 1. Entrevista final].

Finalmente, en la Figura 3 también se observa que una maestra indica que a ella siempre le ha gustado impartir esta disciplina porque lo considera «un contacto diferente con el alumno (...) juegas con ellos, no sé, veo que es positivo» [Maestra 2. Grupo de discusión].

\section{Discusión}

A lo largo de esta investigación han aparecido diversos resultados que, tras su análisis, dan respuesta a los objetivos planteados en la misma. En este sentido, si se atiende al primer objetivo planteado, «analizar el pensamiento docente sobre la implementación de los espacios de aprendizaje y su incidencia en el proceso de aprendizaje durante las sesiones de Educación Física Infantil», se puede señalar lo siguiente. La opinión docente indica que los EA tienen una repercusión positiva en el alumnado independientemente de las capacidades o competencias cognitivas que posea cada uno, como indican los estudios de Rachel et al. (2016) y los de RimmKaufman y Sandilos (2015).

Se observa que las maestras opinan que los juegos propuestos y realizados en los EA suponían un reto para el alumnado ya que por su grado de complejidad eran más difíciles de lo habitual, lo que hacía crecer la motivación del alumnado considerablemente, lo que ya señalaban García y Alarcón (2011) en su estudio.

Por otro lado, del pensamiento docente se deduce que los EA favorecen el desarrollo emocional del alumnado, ya que ha habido alumnos que han demostrado su alegría y entusiasmo hacia estos espacios, mientras que también se ha podido observar el miedo y rechazo de una niña sufrió hacia el rincón de los disfraces. Este hecho contrasta en positivo con los estudios de Lagardera y Masciano (2014) dado que estos indican que el desarrollo de la conducta motriz en el alumnado implica el progreso de las emociones personales, o el estudio de Brown (2014) al señalar que los EA generan en el alumnado el desarrollo de su propia personalidad mediante el desarrollo de emociones y sentimientos.

Cabe considerar por otra parte, que los estudios de Burghardt (2014) y Lavega et al. (2014) indican que la conducta motriz es capaz de favorecer el desarrollo de las dimensiones cognitiva, social, afectiva y biológica. En este sentido, la vivencia de las maestras participantes en esta investigación sugiere que mediante el uso de estos EA el alumnado parecía presentar más relaciones sociales e interpersonales entre los compañeros que en otro tipo de sesiones más dirigidas y con menos margen para la toma de decisiones.

Respecto al segundo objetivo, «examinar la repercusión de los espacios de aprendizaje sobre la práctica docente: ideas de las maestras, sentimientos generados, esfuerzo realizado, y propuestas de cambio o mejora, entre otros», se pueden señalar varios aspectos.

Un resultado destacable es comprobar el cambio del pensamiento docente respecto a la idea preconcebida de que los EA generarían mucho descontrol durante la sesión de EF. Ellos creían que el alumnado causaría mucho alboroto en el pabellón y que no sería capaz de jugar con los materiales cumpliendo las normas establecidas. Esta buena actitud por parte del alumnado y la aceptación de estas metodologías durante las sesiones de EF pudiera deberse a que los EA atraen la atención del alumnado (Blández, 2000) y provocan una actitud positiva y adecuada de estos.

Por otro lado, los docentes señalaron que es necesario que los alumnos se liberen de vez en cuando de las actividades muy dirigidas en el aula y que están definidas previamente, algo que también señalan los estudios de Fajardo y García (2010) y de Navarro y García (2010).

Otro resultado de esta investigación se centra en la capacidad que las maestras han tenido para aplicar las metodologías empleadas durante el programa de intervención en sesiones de EF Infantil ajenas al mismo. Se sospecha por tanto que se ha conseguido enriquecer la práctica docente de las maestras con el empleo en el aula de los EA. Esto sería en sí mismo un cambio realizado por las maestraspara mejorar el aprendizaje del alumnado y el desarrollo de sus capacidades, como señalan algunos estudios de Blackmore et al. (2011) y de Walker et al. (2011).

Finalmente, el uso de los EA genera a su vez resultados positivos tanto en docentes como en alumnos, como también muestran los estudios de Navarro y García (2010) y Fajardo y García (2010). Esto se comprueba al observar el contacto que se da entre ambas figuras, un contacto más estrecho y cercano que con el desarrollo de otras metodologías en las sesiones de EF Infantil, como indican las participantes. No se trata de sentenciar que los EA favorecen siempre y en cualquier caso el mantenimiento de una relación positiva entre los alumnos y los docentes, pero sí se puede señalar que parece haber 
tendencia hacia ello en este caso.

\section{Conclusiones}

Los EA son una forma diferente de generar el desarrollo integral del alumnado mediante el juego motor y la actividad física. Debido a la naturaleza más libre y menos dirigida de este tipo de espacios, los alumnos experimentan una mayor motivación al sentir que son ellos quienes deciden sus actos. Estos espacios invitan al aprendizaje mediante la estimulación del alumnado, además en ellos se pueden generar diferentes conductas motrices que invitan al desarrollo emocional de la persona, así como al desarrollo de los aspectos sociales y cognitivos.

Los docentes han sido capaces de observar que el alumnado está muy motivado con esta metodología y que es una forma diferente de fomentar el desarrollo global del individuo, por ello fueron varias las veces las que volvieron a utilizar estos EA en el aula, más allá de los realizados para la presente investigación. Con esto se comprueba que los EA no solo motivan al alumnado, sino también a los docentes.

En definitiva, los EA son otra forma de enriquecer el proceso de enseñanza y aprendizaje, por lo que se han de tener en cuenta en las programaciones docentes e incluirlos en las sesiones de EF Infantil.

\section{Referencias}

Abbott, J. (2015). Designing 1-to-1 Physical Learning Spaces for Contemporary Learners. First let's talk about the learning, then we'll talk about technology. Layers of Teaching and Learning.

Adolph, K.E. \& Franchak, J.M. (2016).The development of motor behavior. Wiley Interdisciplinar Review Cognitive Science, 8(1-2). DOI: 10.1002/wcs. 1430.

Arufe, V. (2020). ¿Cómo debe ser el trabajo de Educación Física en Educación Infantil? Retos, 37, 588-596. DOI: https://doi.org/10.47197/ retos.v37i37.74177.

Ates, H. (2013). Information Technology and The Learning Environment in Primary School. Procedia. Social and Behavioral Sciences, 93, 695-698. DOI: 10.1016/j.sbspro.2013.09.263.

Barrett, P., Davies, F., Zhang,Y. \&L,B.(2015).The impact of classroom design on pupils' learning: Final results of a holistic, multi-level analysis. Building and Environment, 89, 118-133. DOI: 10.1016/ j.buildenv.2015.02.013.
Bássoli, A.A., Gonçales, D.M. \& Matias, V.F. (2021). O movimento como porta de acesso para a aprendizagem. Retos, 41, 834-843. DOI: https:// doi.org/10.47197/retos.v41i0.84287.

Blackmore, J., Bateman, D., Loughlin, J., O’Mara, J. \& Aranda, G. (2011). Research into the connection between built learning spaces and student outcomes. Literature Review, 22.

Blández, J. (2000). Programación de unidades didácticas según ambientes de aprendizaje. Barcelona, España: Inde.

Bisquerra, R. (2004). Metodología de la investigación educativa.Madrid: La Muralla.

Brown, F. (2014). Play and Playwork. Maindebnhead, Inglaterra: McGraw-Hill Education.

Burghardt, G.M. (2014). Play, playfulness, creativity and innovation. Patrick Bateson and Paul Martin. American Journal of Play, 6(3), 411-413. DOI: 10.12966/ abc.05.02.2014.

Corral, Y. (2009). Validez y confiabilidad de los instrumentos de investigación para la recolección de datos. Revista Ciencias de la Educación, 19(33), 228-247.

Fajardo, M.T. \& García, J.J. (2010). El empleo de ambientes de aprendizaje en Educación Física, para la enseñanza del equilibrio en primer curso de Educación Primaria. II Jornadas de los Máster en Investigación e Innovación en Educación Infantil y Educación Primaria, pp.65-84. Murcia, España: Universidad de Murcia.

Flick, U. (2007). Introducción a la investigación cualitativa. Madrid: Sage.

Duncan, P \& Arnott, L (2019). Exploring the pedagogic culture of creative play in early childhood education. Journal of Early Childhood Research, 1-30. DOI: https: / /doi.org/10.1177/1476718X19867370.

García, E. \& Alarcón, M.J. (2011). Influencia del juego infantil en eldesarrollo y aprendizaje del niño y la niña. Revista digital efdeportes.com, 153.

Gil-Espinosa, F.J., Romance, Á.R. \& Nielsen, A. (2018). Juego y actividad física como indicadores de calidad en Educación Infantil. Retos, 34, 252-257. DOI: https: / /doi.org/10.47197/retos.v0i34.60391.

Hernández, A., González, I., Sánchez, Y. \& Carrión, S. (2020). Los ambientes de aprendizaje en Educación física y motivación en las primeras edades. Retos, 38, 761-767. DOI: https://doi.org/10.47197/ retos.v38i38.77441.

Hyndman, J., Kevitt, T. L. \& Mc, P. (2012).AmbiLearn:Enhancing the Learning Environment for Primary School Education. En Proceedings of the Third international conference on Serious Games Development and Applications (pp.231-242). Berlin,Ale- 
mania: Springer.

Iglesias, M.L. (2008). Observación y evaluación del ambiente de aprendizaje en Educación Infantil: dimensiones y variables a considerar.Revista Iberoamericana de Educación, 47.

Jung, J. (2011). The focus, role, and meaning of experienced teachers' reflection in physical education. Journal Physical Education and Sport Pedagogy 17(2), 157-175.

Lagardera, F. \& Lavega, P. (2011). Educación Física, conductas motrices y emociones. Revue ethologie \& praxéologie, 16, 23-43.

Lagardera, F. \& Masciano, A. (2014). La pedagogía de las conductas motrices en el gimnasio Olimpia de Chivilcoy. Revista Iberoamericana de Ciencias de la Actividad Física y el Deporte, 31(1), 34-45.

Lavega, P., Planas, A. \& Ruiz, P. (2014). Juegos cooperativos e inclusión en educación física. / Cooperative games and inclusion in physical education. Revista Internacional de Medicina y Ciencias de la Actividad Física y el Deporte, 14(53), 37-51.

López-Pastor, V.M. (2006). Una forma de entender y trabajar la motricidad en Educación Infantil. Seminario de la E.U. de magisterio de Segovia. Planteamientos básicos.La Peonza. Revista de Educación Física para la Paz, 1, 3-9.

McMillan, J.H. \& Schumacher, S. (2011). Investigación educativa. Madrid, España: Pearson. Addison Wesley.

Meneses, M. \& Monge, M.A. (2001). El juego en los niños: enfoque teórico. Revista Educación, 25(2), 113 124.

Miraflores, E \& Goldaracena, I. (2021). Análisis de la psicomotricidad a través de la práctica psicomotriz de Bernard Aucouturier: estudio de casos. Retos, 39 , 620-627.

Montero, M.G. (2012). Diseño e implementación de 4 rincones de aprendizaje para niños de 4-5 años con una guía de apoyo para el docente. Trabajo de graduación previo a la obtención del Título de Licenciada en Ciencias de la Educación, mención: Educadora Especial y Preescolar. Cuenca, Ecuador: Universidad de Azuay.

Moreno, J.A., López, B., Gutiérrez, E.M., Cascada, M. \& Fernández, M.R. (2004). Situación actual de la motricidad en la etapa de 0 a 6 años según el profesorado de Educación Infantil. Revista Iberoamericana de Educación Física y Tendencias Corporales, 16, 17-34.

Nielsen, A., Romance, Á.R. \& Chinchilla, J.R. (2020). Los ambientes de aprendizaje como metodología activa promotora de la actividad física en Educación Infantil. Un estudio de caso. Retos, 37, 556-562.
Navarro, B. \& García, J.J. (2010). El uso de ambientes de aprendizaje en Educación Infantil para trabajar contenidos de Educación Física. II Jornadas de los Máster en Investigación e Innovación en Educación Infantil y Educación Primaria, 399-416. Murcia, España: Universidad de Murcia.

Parlebas, P. (2001). Juegos, deporte y sociedad. Léxico de la praxiología motriz.Barcelona, España: Paidotribo.

Rachel, A., Nnamdi, O. \& Thomas, I. (2016). Learning Environments as Basis for Cognitive Achievements of Students in Basic Science Classrooms in Nigeria. Universal Journal of Educational Research 4(6), 1471 1478. DOI: 10.13189/ujer.2016.040627

Rimm-Kaufman, S. \& Sandilos, L. (2015). Improving Students'Relationships with Teachers to Provide Essential Supports for Learning. International Multidisciplinary E-Journal, 5(9), 46-49.

Sáenz-López, P. (1999). La importancia de la Educación Física en Primaria. Revista digital Apunts. Educación Física $y$ deportes, 57, 20-3.

Smith, PK. \& Pellegrini, A. (2013). Learning Through Play. En RE. Tremblay, M. Boivin y RDeV. Peters. (Eds.), Encyclopedia on Early Childhood Development [online].

Uncapher, M. (14 de octubre, 2016). The Science of Effective LearningSpaces. [Post en un blog].Recuperado de: https: / /www.edutopia.org/ article/science-of-effective-learning-spaces-meli-nauncapher.

Velasco, V. \& Díaz de Rada, Á. (2006). La lógica de la investigación etnográfica. Un modelo de trabajo para etnógrafos de escuela. Madrid, España: Trotta.

Vicente, F. , López, M.A. \& Vallés, C. (2014). Los rincones de trabajo como estrategia en la formación de maestros para la enseñanza de ciencias y su didáctica. Revista Tendencias Pedagógica, 23, 109-126.

Walker, J.D., Brooks, D.C. \& Baepler, P. (2011). Pedagogy and space: Empirical research on new learning environments. Educause Quarterly, 34(4).

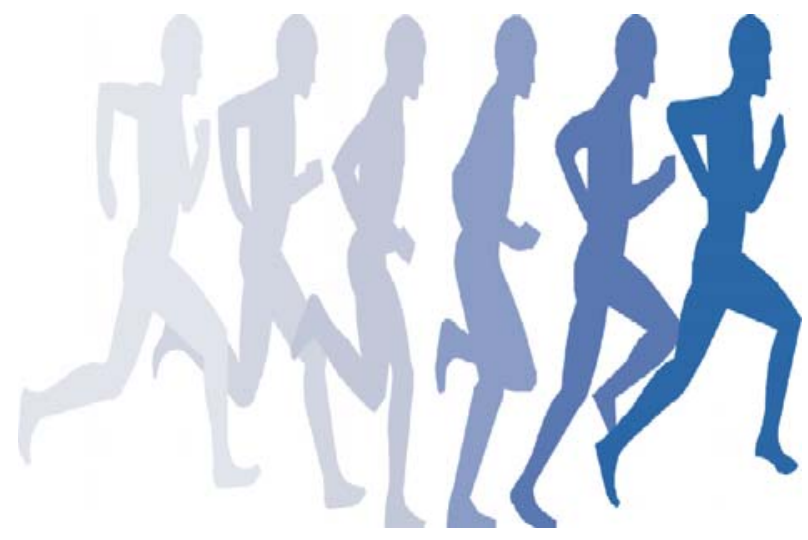

